Mots. Les langages du politique

\title{
Le geste auguste de la République
}

The majestic gesture of the Republic

El gesto augusto de la República

Aude Dontenwille-Gerbaud

\section{CpenEdition}

\section{Journals}

Édition électronique

URL : https://journals.openedition.org/mots/22220

DOI : $10.4000 /$ mots. 22220

ISSN : 1960-6001

\section{Éditeur}

ENS Éditions

\section{Édition imprimée}

Date de publication : 9 mai 2016

Pagination : 49-66

ISBN : 978-2-84788-793-8

ISSN : 0243-6450

\section{Référence électronique}

Aude Dontenwille-Gerbaud, "Le geste auguste de la République », Mots. Les langages du politique [En ligne], 110 | 2016, mis en ligne le 09 mai 2018, consulté le 23 avril 2022. URL : http:// journals.openedition.org/mots/22220; DOI : https://doi.org/10.4000/mots.22220 


\section{Le geste auguste de la République}

Maurice Agulhon évoque, à propos de la période de fondation de la République, une véritable lutte de symboles dans l'espace public (Agulhon, 1988, p. 108). Durant le Second Empire, l'Église catholique a multiplié les sanctuaires ou les Vierges en majesté (comme au Puy-en-Velay où le monument dû à Jean-Marie Bonnassieux atteint $16 \mathrm{~m}$ de haut, plus de $22 \mathrm{~m}$ avec le piédestal). L'espace est donc à laïciser. Cette lutte symbolique n'est pas complètement nouvelle, elle date des années 1830, mais elle s'amplifie grandement. Au sein de ce que d'aucuns nommeront "statuomanie », un geste récurrent retient notre attention : le bras levé horizontalement, index pointé vers l'horizon.

Il ne faut certes pas négliger le conservatisme général de la statuaire publique et particulièrement du monument commémoratif à la fin du $19^{\mathrm{e}}$ siècle : un même type, un modèle, une allure générale et surtout la volonté de réalisme. Pour autant, nous formulons l'hypothèse que ce geste n'est pas de l'ordre de la gestuelle des personnages, mais bien d'une volonté identitaire politique, geste démonstratif et revendiqué, très largement repris, y compris dans les caricatures, véritable mise en scène du projet républicain. Cette théâtralité est aussi celle de l'espace dans lequel s'effectue ce geste. Comme l'analyse Antoine Prost, un monument ne peut se réduire à sa statuaire, il forme un tout (Prost, 1984, p. 200). Il devient, au fil du temps, lieu de mémoire, ces lieux «simples et ambigus, naturels et artificiels, immédiatement offerts à l'expérience la plus sensible et, en même temps, relevant de l'élaboration la plus abstraite» (Nora, 1984, p. xxxIV). Anne-Claude Ambroise-Rendu interroge ce qu'est le geste en politique :

Dans une définition rapide, posons qu'il est un moment suspendu, un mouvement interrompu, reconnu pour lui-même, préparé, ou qui fait irruption par inadvertance (hasard?), séparé de ce qui le précède, et de ce qui lui succède. Peu importe la durée, c'est une fraction de temps qui se distingue du mouvement continu, qui s'isole et se charge de sens. (dans Ambroise-Rendu et al., 2006, p. 194)

Le geste de la statue, au sein d'un monument s'inscrivant lui-même dans l'espace urbain, les éléments linguistiques qui parfois l'accompagnent, les 
paroles prononcées lors des inaugurations de ces monuments, les commentaires qu'en fait la presse forment un vaste discours que nous proposons d'analyser comme partie intégrante de l'événement fondateur de la Troisième République. Dans une perspective d'analyse du discours, cette diversité d'actes langagiers peut s'interroger à l'aune du concept développé par Jacques Guilhaumou (2006), qui reprend les catégories introduites par Reinhart Koselleck d'espace d'expérience et d'horizon d'attente (traduit en 1990). Ce vaste discours tisse en effet un lien indéfectible entre l'expérience révolutionnaire et l'horizon d'une République sociale Une et Indivise pour un peuple de citoyens libres. Plusieurs trajets thématiques, imbriqués les uns dans les autres, traversent ces «événements », interrogeant la guerre et la paix, le désordre et l'ordre, l'obscurantisme et les lumières de la science, la ou les questions sociales. La présence de cette statuaire dans l'espace public, acte de transmission, réalise un possible de cet horizon d'attente par ses inaugurations, commémorations, commentaires : se montrer homme régénéré donc libre, digne du suffrage universel, tout en héritant du bouillonnement des actes fondateurs de la Révolution. Interroger cet événement discursif, constitué tout à la fois d'un geste et d'une parole, nécessite de préciser les pistes méthodologiques qui permettent d'analyser cet acte de langage. Nous nous référerons aux principes énoncés par François Rastier à propos de la sémiotique des cultures (Rastier, 2009, p. IX) : l'objet de la sémiotique n'est pas fait de signes mais de performances complexes qui constituent le milieu humain, le monde où nous vivons. Toute pratique sociale met en jeu des performances sémiotiques multiples (Rastier, 1996, p. 241) La sémiologie interprétative développée par François Rastier peut ainsi rendre compte de la polysémie de monuments porteurs de valeurs tout autant éthiques qu'esthétiques. D’un point de vue méthodologique, la caractérisation des signes dépend donc des parcours interprétatifs. La seule «description » d'un monument n'a de sens que située dans la démarche herméneutique du chercheur. Nous mobiliserons également le concept d'interdiscours. Marie-Anne Paveau nous met en garde à propos de l'instabilité chronique de cette notion, souvent considérée comme analogie de l'intertextualité (Paveau, 2010, p. 7). C'est bien ici l'interdiscours comme espace de conflits et de contradiction - concept hérité du matérialisme historique et de la psychanalyse lacanienne -, que souhaite interroger l'historienne. En décrivant le geste du bras et de l'index tendus de la statuaire républicaine, nous nous efforcerons de montrer comment, au fur et à mesure de notre analyse, nous nous situons dans une forme de convergence métadiscursive.

Une telle analyse ne peut se mener que dans une dimension contrastive et comparative. Nous aurons donc à préciser la spécificité de ce geste du bras par rapport à celui de la statuaire - et plus généralement de l'iconographie -, d'autres périodes historiques (sculptures antiques, Renaissance, moderne, iconographies religieuses, monarchistes, bonapartistes...). Notre corpus sera 


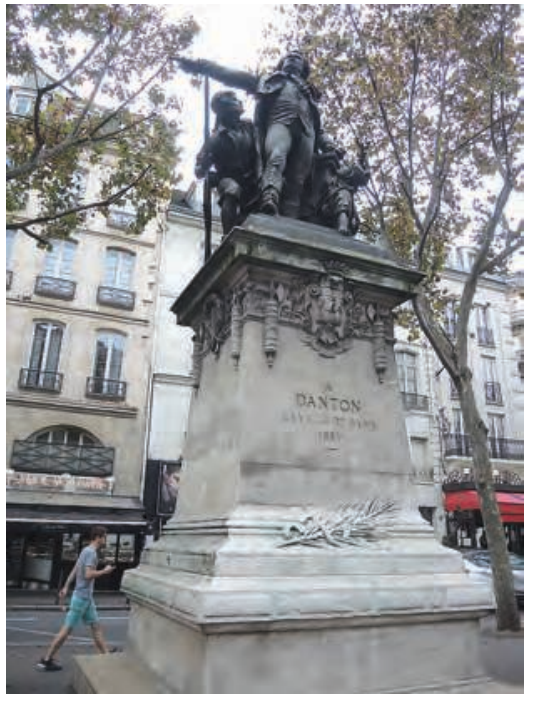

Figure 1.

Monument Danton, 1891, par Auguste Paris. Carrefour de l'Odéon, Paris 6e. (c) Aude Dontenwille-Gerbaud.

ainsi constitué d'une dizaine de monuments, dont certains n'existent plus aujourd'hui et qu'une recherche par cartes postales permet de mettre au jour. Il sera complété par un corpus d'œuvres picturales, quelques caricatures, des discours tenus lors des inaugurations et des articles de presse. La période couverte s'étend largement de la Révolution française à 1914. Enfin, nous étudierons plus précisément l'un de ces monuments, celui à la gloire de Léon Gambetta érigé en 1888 place du Carrousel à Paris. Cette étude ne se veut nullement exhaustive et il ne s'agit ici que d'explorer et d'étayer une hypothèse. Une analyse plus systématique de la statuaire républicaine serait seule en mesure d'en administrer la preuve, au sens historien du terme.

\section{Polysémie d'un geste qui s'inscrit dans l'histoire}

La statuaire l'a saisi et traduit après sa mort dans le geste que l'imagination lui attribue et qui est devenue classique. Mais si elle l'a traduit dans la statue de la place du Carrousel, on peut dire qu'elle l'a trahi, en lui donnant un geste théâtral, forcé, ampoulé, faux, absolument contraire à sa pensée et à son mot [...]. De l'œuvre et de l'homme, il ne reste plus aujourd'hui qu'un geste..., malheureusement pour lui, malheureusement pour nous. (Hacks, 1892, p. 94)

Ainsi s'exprime Charles Hacks, auteur d'un ouvrage, en 1892, dit de « sémiologie » et intitulé Le geste. Cet essayiste, ancien médecin et aventurier, s’inscrit dans le courant antiparlementaire qui se développe à l'époque du scandale de Panama. Pour lui, le geste s'oppose à l'acte, la mimique à l'action (Deloye, 


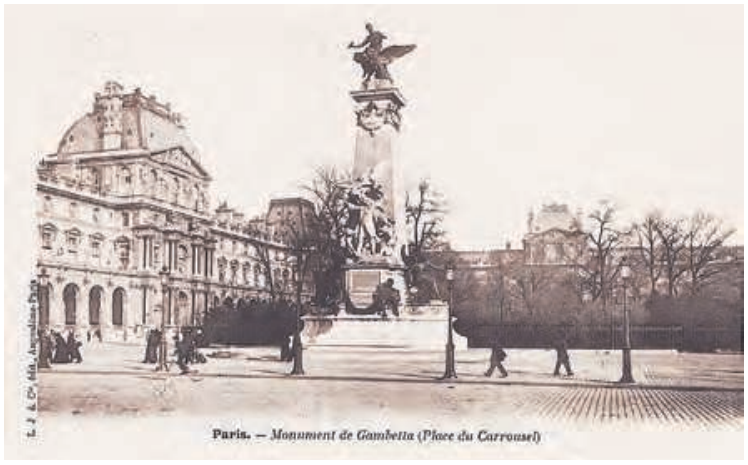

Figure 2.

Monument Gambetta, 1888, par Jean-Paul Aubé, sculpteur, et Louis-Charles Boileau, architecte.

Carte postale, L. J. \& Cie édit., Angoulême-Paris.

1992, p. 129). Par ailleurs, Ch. Hacks se veut fondateur d'une science nouvelle, la sémiologie du geste, « objet mal défini pour une science sans nom », selon AnneMarie Drouin (1992). Le geste du monument Gambetta - ce dernier représenté le bras droit tendu et un index pointé - apparaît au publiciste emblématique des dérives de la Troisième République. À l'inverse, dans une monographie dédiée au monument, l'architecte décrit ce même geste comme la représentation symbolique du combat victorieux de la République (Boileau, 1886).

\section{Le geste de l'orateur}

Ce geste renvoie de façon classique au geste antique de la declamatio. Dès Cicéron et Quintilien, ce geste se veut le signe d'une pensée ou d'une volonté qui s'impose (Cicéron, 1966-1985; Quintilien, 1975-1980). En cette toute première approche, le geste ici décrit n'aurait rien de particulièrement emblématique de la Troisième République. Il ne ferait que s'inscrire dans une longue tradition rhétorique que la sculpture a elle-même contribué à codifier. Le geste du monument Gambetta (1888) se retrouve dans la statuaire de Danton (1891). Il était déjà présent dans une sculpture plus ancienne, celle de Mirabeau par François Truphème (1870) que l'on peut toujours observer dans la salle des «pas perdus» du Tribunal d'Aix-en-Provence.

II n'est plus possible, en revanche, d'analyser comme geste d'orateur celui du Monument aux Enfants de l'Eure-et-Loir (Burollet, 1989, p. 95) ou celui du Monument aux Enfants du Rhône.

\section{Le geste guerrier}

Ce même geste, bras tendu (mais ici bras gauche), index levé, est celui d'allégories qui renvoient cette fois davantage au geste antique d'ad locutio des empereurs romains. Il s'agit là d'un signe guerrier, celui des harangues militaires, et non plus d'un geste d'assemblée. De ce point de vue, le geste de la 


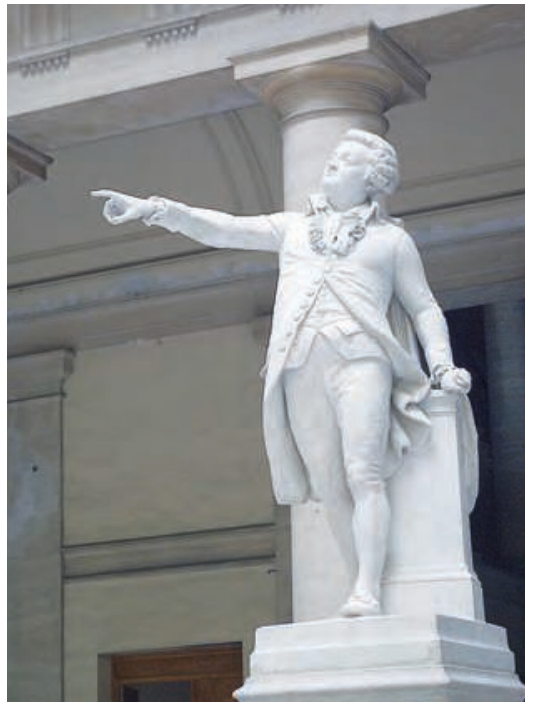

Figure 3. Mirabeau, 1870, par François Truphème. Palais de Justice d'Aix-enProvence.

(c) Aude Dontenwille-Gerbaud.

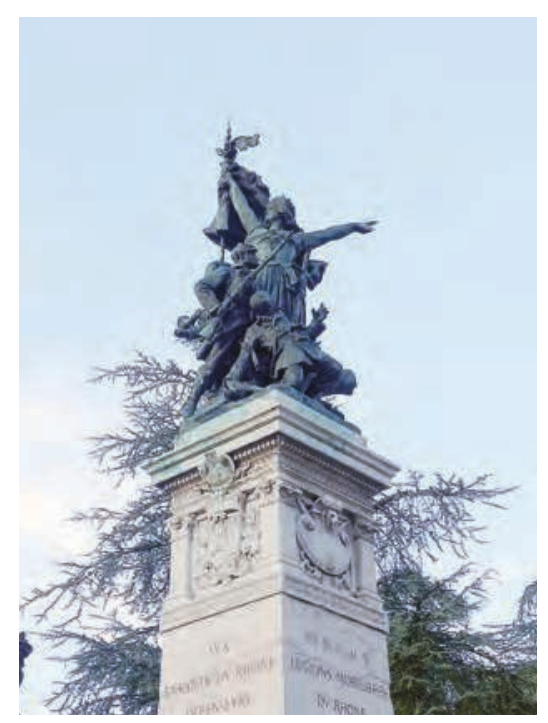

Figure 4. Monument aux Enfants du Rhône, 1887, par Étienne Pagny. (C) Aude Dontenwille-Gerbaud.

statuaire permet de considérer également Danton et Gambetta comme chefs de guerre. S'inscrivant dans l'événement discursif où la presse joue tout son rôle, un commentaire du journaliste de La République française (journal fondé par L. Gambetta), le jour de l'inauguration du monument Danton, en souligne la dimension guerrière :

Il aura fallu près d'un siècle pour qu'un hommage public soit enfin rendu à l'un des plus grands citoyens que notre pays ait produit, à l'un de ceux dont les titres à la reconnaissance nationale sont éclatants entre tous. Qui donc, en effet, a plus fait que Danton pour préserver la France de l'invasion et sauver la Révolution? On peut le dire : sans son initiative hardie, sans son énergie indomptable, sans son action toute puissante, sans son génie politique, le roi, la cour, tous les hommes de l'ancien régime, livraient la France à l'étranger, et la Révolution qui a fait le monde moderne, la Révolution était vaincue. Nous sommes en 1792. Les partis étaient tranchés : d'un côté les patriotes, de l'autre ceux qui trahissaient la France. Cependant l'Assemblée législative tergiversait. Mais le peuple de Paris, Danton à sa tête, sent bien que tout est perdu si l'on n'agit pas sur le champ. Il n'hésite pas et le 10 août, «il enlève à l'invasion la citadelle des Tuileries » et renverse le roi, complice de l'étranger. (La République française, 1891)

La signification guerrière l'emporte alors sur la représentation de l'orateur. Le geste rappelle celui d'Auguste, sculpté en l'an 14 (Augustus - Prima Porta; Rome, musée du Vatican, copie d'un bronze daté de 20 avant J.-C.). Et l'espace 
lui-même fait sens. Le geste de Danton s'oriente à l'Est, vers ces horizons d'où sont venues les troupes étrangères. À Lyon, le geste se déploie également vers l'Est, les provinces perdues à la suite d'une guerre pour laquelle sont morts les «enfants du Rhône».

L'autre bras, toutefois, est à prendre en considération. Gambetta pose son bras gauche sur l'épaule d'un officier. La force du geste du bras droit ne peut se lire sans la force puisée par le bras gauche dans l'engagement au service de la Défense nationale. Celui de Danton s'appuie sur le pupitre de l'Assemblée. La force du geste guerrier se lit/se lie avec celle du geste de l'orateur. Peut-on l'interpréter comme une spécificité emblématique du geste républicain ? Les représentations de Napoléon permettent d'en proposer l'hypothèse. Ce geste, en effet, se retrouve dans le tableau du peintre David, Bonaparte franchissant le col du Grand Saint Bernard, daté de 1800 (Musée national du Château de Malmaison). Le peintre se met alors au service d'un discours officiel, à la gloire du Premier Consul, sauveur de la Révolution française. C'est bien la République qui a voulu la guerre d'Italie. Une fois l'Empire proclamé en 1802, le peintre « officiel» David ne représentera plus l'Empereur bras tendu et index pointé. Alors même que Napoléon $1^{\mathrm{er}}$ s'inscrit dans la lignée des Empereurs romains, le geste d'ad locutio disparaît au profit d'une représentation «en majesté». Le geste du bras ne peut-il s'inscrire que dans l'histoire des temps agités de la conquête républicaine? Nous le retrouvons, en tout cas, dans une iconographie célèbre de la période révolutionnaire, celle de la danse de la Carmagnole (Eau forte anonyme, 1792, Musée Carnavalet, Paris). Un sans-culotte désigne de son index, bras tendu, l'ennemi vaincu, geste tout aussi guerrier que politique puisque c'est dès lors du côté du «peuple», d'en bas, que s'effectue ce geste (Garrigues, 1988, p. 36).

\section{Le geste de quel pouvoir?}

Ce geste, en effet, n'existe pas seulement en lui-même mais par celui qui s'en empare. La statuaire républicaine se différencie alors clairement de celle de l'Ancien Régime par la mise en scène de "grands hommes". Il est aussi à mettre en relation avec l'évolution des publics. Jusqu'au $18^{\mathrm{e}}$ siècle, l'iconographie en général, la statuaire en particulier, s'appuie sur une tradition cultivée, la culture humaniste. Elle se simplifie sous la Révolution pour s'adapter au nouveau public visé. Bernard Richard analyse comment nombre de conventions se modifient avec le temps, comment un nouvel usage d'un symbole donné par les acteurs politiques suggère une nouvelle perception (Richard, 2012, p. 18). Il insiste sur la vogue de la statuaire en lieu public : chaque année, l'Almanach Hachette, très lu et s'efforçant à l'exhaustivité, publie la liste des nouveaux monuments qui «décorent la France» (ibid., p. 341). Le contre-pied de cette vogue s'exprime par la grande tradition de dénigrement de cette sta- 
tuaire, laquelle ne concerne pas seulement l'opposition conservatrice et d'extrême gauche. L'avant-garde artistique (Rodin, Maillol...) ridiculise ouvertement ce type d'expression (Agulhon, 2004, p. 18). Avant 1882 et la fondation de la Société des artistes français, le monopole revient au Salon et à l'art dit « officiel» (Genet-Delacroix, 1985, p. 116). Il faut attendre 1903 pour voir se dégager la notion de «propriété intellectuelle» et donc de liberté créatrice. Malgré les attaques très vives contre l'autorité considérable de l'École des BeauxArts et de l'Académie de France à Rome, hostiles à tout courant nouveau (Pingeot, 1986a, p. 28), les artistes désireux d'évoquer le monde contemporain ne peuvent guère se le permettre que par des formes symboliques ou allégoriques se mêlant au réalisme de la composition. Que peut représenter ce geste du bras tendu et de l'index pointé, entre nécessité de s'inscrire dans un «art officiel» et recherche d'une certaine originalité et autonomie créatrice?

Prenons l'exemple de la fresque de la galerie Saint-Louis de la Cour de Cassation à Paris (Roze, 1990, p. 39). Le Saint-Louis peint par Luc Olivier Merson en 1877 est représenté rendant la justice à son retour de terre sainte, bras droit tendu presque horizontalement. On pourrait y voir une référence classique aux représentations médiévales et Renaissance présentes dans les vitraux : basilique Saint-Denis, abbaye de la Trinité de Fécamp, chapelle du château de Champigny-sur-Veude par exemple, ou encore celles des miniatures: Vie et miracles de Saint-Louis par Guillaume de Saint-Pathus, Heures de Jeanne d'Évreux ou de Jeanne de Navarre (Teisseyre, 1977, p. 409-414). Toutefois, ce geste est classiquement dirigé vers le bas ou vers le haut et non horizontalement; le plus souvent, ce sont index et majeur groupés qui sont pointés. Vers le bas, le geste symbolise la justice royale rendue pour les sujets. Vers le haut, le geste symbolise la justice royale d'essence divine. Or, Luc Olivier Merson, qui répond à de nombreuses commandes publiques depuis son Premier Prix de Rome en 1869 (on lui doit, au Sacré-Cœur de Paris, la fresque recouvrant l'abside, mais également des fresques à la Sorbonne, à l'Hôtel de Ville, à l'OpéraComique...) choisit, en 1877, le geste du bras droit tendu horizontalement, index pointé. Émile Zola avait salué dès 1875 les «compositions énigmatiques» du jeune Merson, qui réussissait toujours à faire entendre dans ses décors officiels une note bizarre, une couleur qui étonne (Stévenin, Ribemont, 2009). La sémiotique des cultures permet ici d'analyser cette complexité. Selon le contexte, le même signe fonctionne comme un indice, index, symbole, etc. (Rastier, 2008, p. 6) La culture est ici celle des magistrats de la Cour suprême, qui se veulent héritiers des Parlements d'Ancien Régime. Dans la galerie de la Cour de Cassation, institution créée par la Révolution elle-même (décret des 27 novembre et $1^{\text {er }}$ décembre 1790), les magistrats peuvent contempler un geste évoquant, certes, la justice d'Ancien Régime, mais celui-ci est réinterprété pour une nouvelle identité républicaine. 


\section{L'horizon d'attente républicain}

Au musée Carnavalet à Paris, une gravure anonyme du $18^{\mathrm{e}}$ siècle place en regard une allégorie de la Liberté (ou une représentation symbolique de la Constituante de 1790) et le Pape. Ce dernier, sur un trône, souffle des «bulles » (Pariset, 1989, p. 225). La Liberté, assise sur la Constitution, en surplomb du Pape, portant fleurs de lis et couronne, tend son bras droit et pointe de l'index les bulles. Elle tient du bras gauche les faisceaux surmontés du bonnet phrygien et s'adosse à la Déclaration des Droits de l'Homme. L'index semble vouloir crever les bulles. Le pouvoir de l'Église est alors défié par ce geste de revendication révolutionnaire. Nous pouvons mettre en regard de cette gravure du $18^{e}$ siècle d'autres bras tendus et index pointés qui défient le pouvoir de l'Église. La statue de Lavoisier (œuvre aujourd'hui disparue de Louis-Ernest Barrias inaugurée en 1900), qui se trouvait à Paris derrière la Madeleine, face à la rue Tronchet dans le $8^{e}$ arrondissement, tend le bras droit et pointe l'index dans l'alignement du geste du bras gauche qui s'appuie, lui, sur une table où reposent des instruments de mesure. Que pointe l'index, si ce n'est le progrès scientifique? On retrouve ce même geste dans le monument en hommage à François Arago à Perpignan, où le geste du personnage, bras droit tendu et index pointé, poursuit l'axe initié par le globe situé à l'arrière.

Est célébré ici tout autant le savant astronome et mathématicien que l'homme politique de 1848. Mais c'est aussi l'orateur et le pédagogue, capable de donner un cours d'astronomie à un public populaire. L'index pointe l'horizon d'attente d'une République fondée sur la science et porteuse de progrès. En ce sens, le geste répond aux gestes identitaires de nature religieuse. Dans les Annonciations médiévales, l'ange dirige son bras droit et son index tendu, la parole divine se déployant dans ce geste performatif. Jean Arrouye l'analyse pour une Annonciation anonyme de 1340 du musée Granet d'Aix-enProvence (Arrouye, 1998, p. 25-39). Et comment ne pas penser à la Chapelle Sixtine et au double geste représenté par Michel-Ange ? L'index d'Adam (bras gauche) pointe le doigt de Dieu (bras droit), geste toutefois ambivalent. Comme le montre Daniel Arasse, l'index pointé jalonne l'œuvre du peintre et sculpteur et fonctionne comme un signe à insérer dans une série (Arasse, 1981, p. 19). Se référant à Julia Kristeva, D. Arasse évoque la gestualité comme une pratique, comme l'élaboration d'un message. Il part de la signification conventionnelle du geste pour mettre au jour l'obscurcissement relatif de cette signification. Contrairement aux représentations de Giotto, les index d'Adam et de Dieu par Michel-Ange ne se touchent pas, ils sont infiniment séparés, ce qui peut se lire comme une fêlure, celle de la séparation. Le geste de François Arago (bras droit), geste de scientifique qui n'a plus besoin de Dieu, semble reprendre des codes sculpturaux qui s'inscrivent bien évidemment dans l'histoire de l'esthé- 


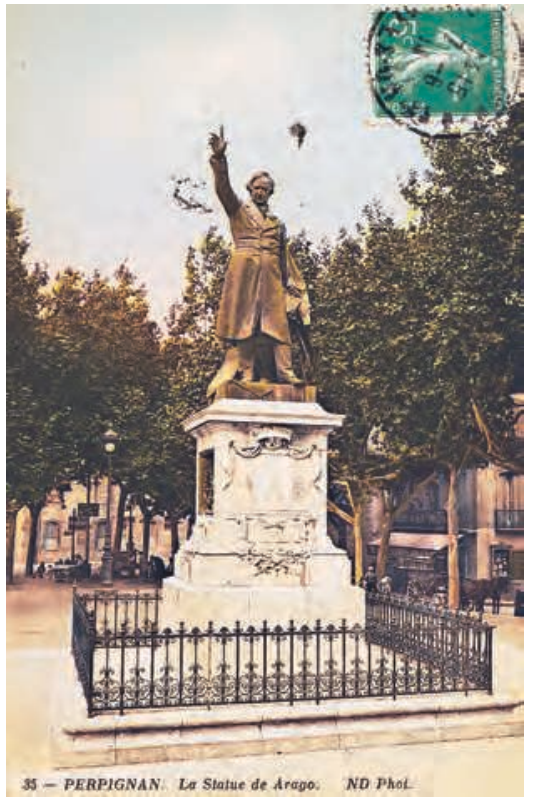

Figure 5. Monument François Arago, 1879, par le sculpteur Antonin Mercier. Socle de Paul Pujol, architecte (Carte postale ND Phot).

tique, tout en en détournant le sens. Il est dès lors possible de faire dialoguer deux époques et deux esthétiques pour en conclure que ce geste du bras tendu et de l'index pointé prend une dimension tout à la fois philosophique et politique. Le triomphe des héros culturels représente les idéaux des Lumières et les valeurs de l'Éducation (Hargrove, 1986, p. 256). La lecture classique, considérant la statuomanie républicaine comme un ensemble de représentations naïves, simple forme de propagande du nouveau régime, doit être dépassée si l'on veut restituer à ce projet d'occupation de l'espace des années 18701914 toute sa dimension de projet politique au sein d'un événement discursif.

En 1796, l’Abbé Grégoire l'exprimait ainsi devant le Corps législatif lors de la séance du 11 Pluviôse (an IV) :

Le législateur qui méconnaîtrait l'importance du langage des signes, serait audessous de sa mission; il ne doit laisser échapper aucune occasion de s'emparer des sens, pour réveiller des idées républicaines. Bientôt l'âme se pénètre des objets reproduits constamment sous les yeux; et ce composé, cet ensemble de principes, de faits, d'emblèmes qui retracent sans cesse au citoyen et ses droits et ses devoirs, forment, pour ainsi dire, le moule républicain qui lui donne un caractère national et l'attitude de l'homme libre. (31 janvier 1796)

Nous référant tant à François Rastier (qui refuse de voir fondée la théorie des signes et du sens sur la communication) qu'à Maurice Agulhon (et son projet d'histoire où ethnologie et politique se mêlent), nous pouvons conclure provisoirement : le geste identitaire de cette statuaire publique s'inscrit dans 
un vaste acte de langage, celui des monuments, celui des statues dans le monument, celui des répliques de statue qui ornent progressivement les salons bourgeois, les images qui se diffusent largement sous différents formats, les discours d'inauguration, le travail de l'École qui reproduit ces images dans les manuels scolaires.

Nous pouvons préciser notre analyse à l'aide d'un exemple, celui du monument à la gloire de Léon Gambetta (figure 2, supra), inauguré à Paris, place du Carrousel, en 1888.

\section{Un geste polysémique inscrit dans un espace}

\section{Le monument Gambetta}

Léon Gambetta décède en janvier 1882. Deux ans plus tard, un comité présidé par Eugène Spuller lance un concours pour la réalisation d'un monument à la gloire du tribun. L'interdiscours, au sens d'espace de conflits idéologiques, oppose ici le discours républicain au discours religieux, en fort développement durant le Second Empire et l'Ordre Moral. Il est courant de qualifier les monuments républicains « d'autel de la Patrie », de «cathédrale laïque » et d'insister sur l'importance financière des projets. Le journal créé par Léon Gambetta, La République française, rappelle régulièrement en première page du journal, l'appel à souscription publique. Le crédit est relativement important pour l'époque, l'emplacement du monument est choisi par l'artiste (avant d'être accepté ou non par le conseil municipal), la dimension et le sujet sont libres. Quatre-vingtdeux projets sont exposés à l'École des Beaux-Arts. La République française annonce la composition du jury et invite à la visite de l'exposition. C'est le journal L'Illustration qui fera paraître, le 21 juin 1884 , les reproductions des six projets retenus ${ }^{1}$. Jean-Paul Aubé et Louis-Charles Boileau l'emportent sur d'autres artistes renommés, entre autres Falguière et Dalou. Dans le projet qui emporte les suffrages du jury, c'est finalement la place du Carrousel qui est retenue. Ce monument va ainsi se localiser dans l'espace laissé vide par l'incendie des Tuileries lors de la Commune de Paris, face à l'Arc de Triomphe, tournant le dos à la Cour Napoléon du Louvre. "Le site connote la statue au moins autant que la statue connote le site» (Hargrove, 1986, p. 261). L'interdiscours est aussi celui de la tension entre deux horizons d'attente républicains différents, celui de la République opportuniste et celui de la République sociale. Alors même que la Commune est objet de tabou dans le discours de Gambetta (DontenwilleGerbaud, 2015), choisir de meubler ainsi le «vide» des Tuileries confère à ce monument une valeur symbolique que tout Parisien ne peut ignorer. Sur l'Arc

1. Voir également Hommage à Léon Gambetta, 1982, ministère de la Culture, Délégation aux célébrations nationales, p. 143-144. 
de Triomphe, une autre statue emblématique du projet républicain est pointée par le geste de Léon Gambetta : La Marseillaise de Rude (1833). Comme l'analyse Isabelle Rouge-Ducos, La Marseillaise de Rude est voulue comme un monument dans le monument, une image largement diffusée sur de multiples supports, notamment dans les manuels scolaires (Rouge-Ducos, 2008, p.347). Le geste de Gambetta s'inscrit d'abord dans cet espace, permettant ainsi une véritable lecture de l'histoire : derrière lui, les symboles de l'Ancien Régime et de l'Empire, dans la direction, au bout du doigt pointé, l'hommage à la Révolution française.

\section{Un monument «bavard»...}

Si l'on considère, avec la sémiologie interprétative, que l'activité symbolique est liée à l'imaginaire du corps, si l'on admet que le mouvement est lui-même un fait social de nature historique, ne peut-on considérer ce geste du bras tendu, index pointé, comme un stéréotype discursif (Ducard, 2010, p. 7) ? Nous aurions là un processus de stabilisation sémiotique aboutissant à une forme de schématisme, de figement : Léon Gambetta, en tant qu’homme, disparâ̂trait dans son geste emblématique. Pour reprendre les catégories proposées par François Rastier et Simon Bouquet (2002, p. 243), qui distingue trois zones - dites anthropiques -, de l'entour humain (zone identitaire, zone proximale et zone distale), le geste incarnerait le «Nous» républicain de l'année 1888, le «Vous» du vécu historique entre Révolution et installation de la Troisième République, et enfin, le «Il » de l'horizon d'attente. À la frontière de ces zones, la sculpture fonctionnerait au niveau sémiotique comme objet transitionnel, et le geste comme œuvre codifiée. Le processus, en tout cas, pose la question du rapport entre les différents éléments visuels et les éléments linguistiques du monument. Méthodologiquement, c'est au sein de ce parcours interprétatif que nous utiliserons la description du monument pour caractériser le geste de Léon Gambetta. Partons de la description du haut-relief central proposée par Anne Pingeot :

La diagonale qui donnait son mouvement au groupe passait par le bras de l'orateur qui désignait de son index le drapeau. Gambetta levait la tête pour regarder le Génie de la Patrie, mais c'était lui que fixaient les trois hommes : à droite un officier à demi allongé, son épée brisée, se retenait au fût d’un canon. Gambetta posait la main sur son épaule. À gauche, un marin, un genou en terre, allait saisir un sabre abandonné. Au fond, un simple citoyen en chemise s'était redressé. Il avait saisi un chassepot et s'élançait en se tournant une dernière fois vers l'orateur qui dirigeait ses pas. Le Génie de la Patrie (un jeune homme), survole la composition. (Pingeot, Brese-Bautier, 1986, vol.II, p.14)

Le bras tendu et l'index pointé représentent donc bien tout à la fois le geste de l'orateur - que l'on retrouve à l'envi dans les caricatures - que celui du 
guerrier, puisque Léon Gambetta restera dans l'histoire comme l'homme de la Défense nationale après la proclamation de la République, en pleine guerre contre la Prusse, le 4 septembre 1870. Mais c'est également le geste du député d'Alsace, qui ne cessera de pleurer les provinces perdues. Le groupe de personnages représentés, et l'expression même de Gambetta, amènent l'officier à terre à regardervers l'horizon, celui de la marche, soulignée par la jambe droite de Gambetta qui indique le mouvement. Sur la face centrale de la pyramide, juste au-dessus du haut-relief, quelques lignes datées de septembre 1870 sont inscrites : «Français, élevez vos âmes et vos résolutions à la hauteur des périls qui fondent sur la patrie. Il dépend encore de nous de montrer à l'univers ce qu'est un grand peuple qui ne veut pas périr». Sous le bras droit tendu, toutefois, le personnage qui se redresse n'est ni l'officier ni le marin, mais un simple citoyen. Sa tête est exactement dans le même axe que l'index de Gambetta et la hampe du drapeau, symbolisant ensemble le projet républicain. Ce bras tendu, l'index pointé sont donc d'abord geste politique, celui qui, dès 1869 et le programme de Belleville porté par le leader, annonce la République. Ce geste dirigé vers l'avenir doit se lire dans l'acte de langage que forment tous les grands moments oratoires du tribun, représentation visuelle de "petites phrases» elles-mêmes largement commentées par la presse. Ces formes brèves d'énoncé détachable coproduites par les médias et les politiques (Krieg-Planque, 2011, p. 29) font partie du même événement discursif que le geste de la statuaire. Nous en retiendrons deux, devenues célèbres:

- à Grenoble, le 26 septembre 1872 : «Oui ! je pressens, je sens, j'annonce la venue et la présence, dans la politique, d'une couche sociale nouvelle...» (Gambetta, 1881, III, p.100);

- à Paris, le 15 février 1876 : «Qu'on dise nettement par où il faut commencer, par où il faut continuer et qu'on ne sache jamais par où on doit finir, car le progrès ne s'achève pas, il est indéfini » (Gambetta, 1881, V, p.150).

Le geste de la statuaire pointe l'avènement des couches sociales nouvelles, le progrès infini... Le geste du bras en est le «marqueur». Il désigne aussi la voie à prendre, dans un geste performatif sans ambiguïté : il est de la responsabilité collective, de celle de tout citoyen, de se faire messager de la République. Le geste devient une ferme invitation à partir faire son devoir. Le haut-relief des personnages repose sur un socle. Au revers, le cartouche central indique :

Le 5 janvier 1883, le lendemain des funérailles de Gambetta, ses amis politiques et personnels ont pris l'initiative d'ouvrir une souscription pour élever un monument en son honneur. 19 journaux républicains de Paris et 150 autres de province ont prêté leur publicité. 280000 citoyens français ont souscrit individuellement, d'autres en plus grand nombre ont pris part aux collectes faites en France et dans les colonies françaises. Le Comité a reçu des souscriptions de toutes les colonies de Français en terre étrangère et des dons individuels de beaucoup d'étrangers. 
Le geste du bras tendu et de l'index pointé emporte avec lui ce large espace d'une République devenue puissance coloniale et même puissance mondiale puisque des "étrangers » ont eux-mêmes contribué, désignant ainsi la valeur universelle du message. De fait, tout en haut de la pyramide, la Démocratie, sous la forme d'une femme nue coiffée d'un bonnet phrygien, assise sur un lion ailé, tient la foudre et la Déclaration des Droits de l'Homme et du Citoyen. Si l'on prête attention aux inscriptions, Démocratie est inscrit en gros caractères majuscules, suivie d'un simple «F» comme pour indiquer que la France est porteuse d'un événement qui dépasse largement ses frontières et son histoire propre. Sur les trois autres cartouches, l'on trouve les titres de quelques discours de Léon Gambetta considérés comme emblématiques par le sculpteur. Ce sont là des choix importants qui veulent donner à « lire » le geste lui-même : la Liberté est associée au plaidoyer devenu mythique de Gambetta, jeune avocat de 1868 qui devient dès lors le porte-parole de l'opposition républicaine; la Fraternité est associée au discours de Grenoble de 1872, le «célèbre » discours sur « les couches sociales nouvelles»; l'Égalité est associée au discours parlementaire de 1882 sur l'amnistie des communards. Le lien entre textes et sculpture produit, à l'évidence, un effet de signification voulu par le sculpteur et son commanditaire.

\section{Entre geste de la statue et geste des vivants}

La cérémonie d’inauguration du 14 juillet 1888, largement décrite dans la presse quotidienne nationale, participe de l'acte de langage. Le Petit Parisien, La République française, y consacrent leur Une en retranscrivant in extenso les discours d'Eugène Spuller, l'ami de Léon Gambetta et président du Comité organisateur, ceux de Charles Floquet et Charles de Freycinet. Floquet retient l'origine sociale de Gambetta: "Sorti du peuple, il restera l'honneur du peuple dont il portait en lui les généreux instincts et les grands élans. » Le discours redouble ainsi le geste du bras tendu, insistant sur celui des couches sociales nouvelles, celles de l'avenir. Toutefois, le message tend à se brouiller. Charles Floquet, en effet, s'est battu en duel la veille contre le général Boulanger. La presse, Le Figaro notamment, s’intéresse particulièrement à ce duel en première page du quotidien, éclipsant quelque peu les paroles prononcées par le président du Conseil lors de l'inauguration du monument Gambetta. Un an plus tard, le 14 juillet 1889, le journal satirique La Bombe représentera le général Boulanger en Une, bras tendu et index pointé dans une iconographie intitulée «La prise de la Bastille », réalisée par Paul de Sémant. L’interdiscours matérialise ici un espace de conflits idéologiques multiples entre une République opportuniste triomphante, d'une part, et une double opposition, d'autre part, celle traditionnelle qui refuse encore de considérer l'héritage révolutionnaire et celle, nouvelle, incarnée par l'antiparlementarisme de Boulanger. Le Figaro se montre très critique à propos de la cérémonie d'inauguration du monument. Il 
ironise en effet en page deux à propos du discours prononcé parE. Spuller : «Il a eu des panégyristes en effet pour le mettre sur le pied de Louis XI, Henri IV, du grand Richelieu, du plus grand Danton (sic) », pour en faire un dieu puisque son «monument est un autel, l'autel de la Patrie» (Le Figaro, 14 juillet 1888, p. 2). Le discours d'E. Spuller insiste en fait surtout sur l'avenir, proposant ainsi aux bataillons scolaires qui écoutent le discours à la gloire du grand homme une forme d'exégèse du geste représenté :

Oh! oui, nous le savons bien, nous en sommes heureux, il est là, maintenant et pour toujours, debout, frémissant, éloquent, dans toute l'activité de son ardent patriotisme, obéissant aux inspirations du génie de la France dont il a été tant de fois la voix vibrante, le regard tourné vers l'avenir rayonnant de cette jeune démocratie républicaine. (Spuller, 1888, p. 2)

Le geste du bras tendu et de l'index pointé est ici souligné par le regard. Plus loin dans son discours, le député évoque la « direction » imprimée à la France, les «indications» laissées à la France de l'avenir, la «voie » où tous sont engagés. Que ce soit pour les milliers de spectateurs de l'inauguration ou les lecteurs de la presse, le geste fait sens en cette année 1888. Mais qu'en reste-t-il quelques années plus tard? Qui va encore prendre le temps de « lire » ce monument considéré comme trop «bavard » et critiqué par nombre d'artistes et d'acteurs des pouvoirs publics? Nous ne le saurons jamais. Toutefois, Paul Veyne, analysant la statuaire romaine, a déjà répondu en partie à cette question (Veyne, 1990, p. 7-26). L'historien interroge en effet le fait paradoxal des grands monuments : les détails ne sont guère visibles et de toute façon, difficilement compréhensibles pour l'homme de la rue. Il s'interroge par exemple sur les textes gravés en spirale, tout au long de la colonne Trajane, parfaitement illisibles. II conclut que ces monuments ne sont pas faits pour être lus, que leur valeur est plus expressive qu'informative, que le décor semble ignorer le spectateur dans l'objectif de l'impressionner. Une image agit même si elle n'est pas comprise, ni même regardée... Le vécu quotidien agit par imprégnation silencieuse, conclutil. On peut analyser ainsi le monument Gambetta et le geste du bras, comme emblématiques du projet républicain. Peu importe l'aspect «bavard » du monument. Il n'est là que pour occuper l'espace et montrer un horizon.

Pour conclure, le geste du bras tendu et de l'index pointé, celui d'Arago, Danton, Gambetta, Lavoisier, Mirabeau... s’inscrit bien dans une forme de théâtralité au service d'une expression politique. Le geste ne peut se réduire à une simple "gestuelle», il fait signe au sein d'un vaste discours de ralliement dans les années 1870-1914. Ce choix symbolique s'adresse clairement, durant les années 1870-1879, aux représentants de la réaction conservatrice, à l’Église catholique et ses monuments majestueux. Néanmoins, ce geste vise également une autre forme d'opposition, celle des mouvements socialistes qui commencent à se structurer contre le pouvoir opportuniste, à partir des années 1880. Baude- 
laire, évoquant les statues des places publiques parisiennes, témoigne de leur puissance : "Fussiez-vous le plus insouciant des hommes, le plus malheureux ou le plus vil, mendiant ou banquier, le fantôme de pierre s'empare de vous pendant quelques minutes» (Baudelaire, 1859). L'État de Vichy l'aura bien compris puisque «l'effort de guerre» consistant à utiliser les bronzes prélevés sur les monuments portera d'abord sur les monuments républicains. Pour autant, ce geste perd sens au fil du temps, ce qui pose in fine la question de l'identité collective. Vincent Descombes invite à la prudence avec cette notion (2013, p. 177) et pose philosophiquement la question de la possibilité d'un «nous» historique. Les années passant, le monument Gambetta, par exemple, finira par être démonté en 1954, et les 800 tonnes de pierre entreposées dans les caves du musée d'Art Moderne. À partir de 1965, seul le «bloc Gambetta» sera conservé et le reste envoyé à la décharge. En 1982, pour le centenaire de Gambetta, ce groupe sera installé derrière la mairie du $20^{e}$ arrondissement de Paris, dans le square Édouard Vaillant, où il achève de se détériorer. L'index pointé perd peu à peu ses phalanges! Une maquette donnant la composition d'ensemble se trouve au musée d'Orsay depuis 1980. Les touristes y jettent-ils seulement un regard?

\section{Bibliographie}

\section{Corpus du geste républicain}

Allouard Henri, Monument aux Enfants d'Eure-et-Loir, 1870 (Chartres, aujourd'hui disparu).

Aube Jean-Paul, BoIleau Louis-Charles, Monument à Gambetta, 1888 (Place du Carrousel à Paris, aujourd'hui disparu).

BARRIAS Louis-Ernest, Lavoisier, 1900 (Devant l'église de la Madeleine à Paris, aujourd'hui disparu).

Les Bulles, gravure anonyme du 18e siècle (Musée Carnavalet, Paris).

La Carmagnole, eau forte anonyme, 1792 (Musée Carnavalet, Paris).

DAVID, Bonaparte franchissant le col du Grand Saint Bernard », 1800 (Musée national du Château de Malmaison).

Mercie Antonin, Pujol Paul, 1879, MonumentArago, Perpignan (carte postale ND Phot). PAgnyÉtienne, Monument aux Enfants du Rhône, 1887 (Place du Général Leclerc, Lyon). PARIS Auguste, Danton, 1891 (Carrefour de l'Odéon à Paris).

TruPhème François, Mirabeau, 1870 (Salle des pas perdus, Palais de Justice d'Aix-enProvence).

\section{Pour comparaison}

Annonciation siennoise, 1340 (Musée Granet, Aix-en-Provence). Augustus, Prima Porta, 20 av. J.-C. (Rome, Musée du Vatican). 
BonnASSIEU Jean-Marie, Notre-Dame de France, 1856-1860 (Le Puy-en-Velay).

DEPERTHES Édouard, Urbain II, 1887 (Châtillon-sur-Marne).

Merson Luc Olivier, Saint-Louis, 1877 (Cour de Cassation, Paris).

SaINT-Pathus Guillaume (de), Vie et miracles de Saint-Louis, 1330-1351 (http://gallica. bnf.fr/ark:/12148/btv1b8447303m/f48.item).

\section{Corpus de discours et de presse}

Discours et plaidoyers politiques de Gambetta (1881) rassemblés par Joseph Reinach, Paris, Charpentier (11 volumes).

SPULLER Eugène, 13 juillet 1888, Inauguration du monument Gambetta, Discours, Paris,

Maison Quantin.

L'lllustration : 21 juin 1884.

Le Figaro : 15 juillet 1883, 14 juillet 1888, 14 juillet 1891.

Le Petit Parisien : 14 et 15 juillet 1888.

La République française : juin 1884, juillet 1888, 14 juillet 1891.

\section{Références}

AgulHon Maurice, 1988, Histoire vagabonde, vol.I : Ethnologie et politique dans la France contemporaine, Paris, Gallimard.

- 2004, "Les transformations du regard sur la statuaire publique», La statuaire publique au $19^{e}$ siècle, S. Le Men, A. Magnien éd., Éditions du patrimoine.

Ambroise-Rendu Anne-Claude, D’Almeida Fabrice, EdLEman Nicole, 2006, Des gestes en histoire. Formes et significations des gestualités médicales, guerrière et politique, Paris, Seli Arslan.

ArASSE Daniel, 1981, "L'index de Michel-Ange », Communications, vol.XXXIV, p. 6-24.

ArrouYe Jean, 1998, "Polysémie gestuelle dans une Annonciation siennoise du $14^{\mathrm{e}}$ siècle », Le geste et les gestes au Moyen-Age, CUER MA, Université de Provence, n० 41, p. 25-39.

BaudelaiRe Charles, 2006 [1859], "Le Salon de 1859», La Revue française, Paris, Honoré Champion.

BoIleau Louis-Charles, 1886, Monument à Gambetta. Souscription et programme. Commentaire du projet Aubé-Boileau et Monographie, Paris, Daly fils.

Burollet Thérèse éd., 1989, Quand Paris dansait avec Marianne, 1879-1889, Éditions Paris-Musées.

Deloye Yves, 1992, «Le geste parlementaire. Ch. Hacks ou la sémiologie du geste politique au $19^{\text {e }}$ siècle », Politix, vol. V, no 20, p.129-134.

DONTENWILLE-GERBAUd Aude, 2015, «Les temps héroïques sont clos... L'événement discursif fondateur de la Troisième République, 1870-1881», Cultures des républicanismes, Y. Bosc, R. Dalisson, J.-Y. Frétigné, C. Hamel, C. Lounissi éd., Paris, Kimé, p. 239-251.

Drouin Anne-Marie, 1992, «Un objet mal défini dans une science sans nom. La sémiologie du geste au xIxe siècle », Communications, nº 54, p. 263-287. 
DUCARD Dominique, 2010, «Stéréotypage discursif d'une image de presse » Communication \& langages, $\mathrm{n}^{\circ} 165, \mathrm{p} \cdot 3-14$.

GARRIGUES Jean, 1988, Images de la Révolution. L'imagerie républicaine de 1789 à nos jours, Nanterre, May-BDIC.

Genet-Delacroix Marie-Claude, «Esthétique officielle et art national sous la Troisième République», Le Mouvement social, nº131, M. Rébérioux éd., Les Éditions ouvrières, p. 107-120.

Guilhaumou Jacques, 2006, Discours et événement. L'histoire langagière des concepts, Besançon, Presses universitaires de Franche-Comté.

HACKS Charles, 1892, Le geste, Paris, Marpon et Flammarion.

HARgrove June, 1986, "Les statues de Paris", Les lieux de mémoire, II : La Nation, vol. III, P. Nora éd., Paris, Gallimard (NRF).

- 1989, Les statues de Paris. La représentation des grands hommes dans les rues et sur les places de Paris, Albin Michel.

KoSELLECK Reinhart, 1990, Le Futur passé. Contribution à la sémantique des temps historiques, Paris, EHESS.

KRIEg-Planque Alice, 2011, "Les “petites phrases". Un objet pour l'analyse des discours politiques et médiatiques », Communication \& langages, n 168, p. 23-43.

Magnol-Malhache Véronique, 1996, Léon Gambetta : un saint pour la République?, Caisse nationale des monuments historiques et des sites, Conseil général des Hauts de Seine.

Nora Pierre, 1984, Les lieux de mémoire, vol. I. : La République, Paris, Gallimard (NRF).

PARISET Jean-Daniel, 1989, La naissance de la souveraineté nationale, Paris, Archives nationales.

Paveau Marie-Anne, 2010, "Interdiscours et intertexte... », Linguistique et littérature. Cluny, 40 ans après, Besançon, Presses universitaires de Franche-Comté, p. 93-105.

Pingeot Anne, 1986, La sculpture française au xixe siècle, Paris, Réunion des Musées nationaux.

Pingeot Anne, Brese-Bautier Geneviève, 1986, Sculptures des jardins du Louvre, du Carrousel et des Tuileries, Paris, Réunion des Musées nationaux, 2 volumes.

Prost Antoine, 1984, Les lieux de mémoire, vol. I. : La République, P. Nora éd., Paris, Gallimard (NRF).

RASTIER François, 1996, «Représentation ou interprétation? Une perspective herméneutique sur la médiation sémiotique», Penser l'esprit. Des sciences de la cognition à une philosophie cognitive, , V. Rialle, D. Fisette éd., PUG, 1996, p. 239-259.

- 2008, "Entretien sur les théories du signe et du sens. Réponses à Peer Bundgaard», Texto!, vol. XIII, nº3.

- 2009, Sémantique interprétative, Paris, PUF.

RASTIER François, Bouquet Simon éd., 2002, Une Introduction aux sciences de la culture, Paris, PUF.

RICHARD Bernard, 2012, Les emblèmes de la République, Paris, CNRS.

Roze Jean-Pierre, 1999, La Cour de Cassation. Architecture et décoration, Paris, La Documentation française.

Rouge-Ducos Isabelle, 2008, L'Arc de triomphe de l'Étoile. Art et histoire, Dijon, Faton. 
StÉvenin Anne-Blanche, Ribemont Francis, 2009, L'étrange Monsieur Merson, Musée des Beaux-Arts de Rennes, Catalogue de l'exposition par A.-B. Stévenin, Rennes, Lieux dits.

TEISSEYRE Charles, 1977, «Le prince chrétien aux XVe et XVI ${ }^{e}$ siècles, à travers les représentations de Charlemagne et de Saint-Louis ", Actes des congrès de la Société des historiens médiévistes de l'enseignement supérieur public, Tours, p. 409-414.

VEYne Paul, 1990, «Propagande expression roi, image idole oracle», L'Homme, t.XXX, no 114, p. 7-26. 